\title{
Organization and Implementation of Online Cytology Quality Assurance Program - Georgian Experience
}

\author{
Ekaterina Kldiashvili" (Executive Director, Georgian Telemedicine Union (Association)), \\ Andrejs Romānovs (Associate Professor, Riga Technical University), \\ Nikoloz Shakulashvili (CEO, Georgian Telemedicine Union (Association)), \\ Archil Burduli (Researcher, Georgian Telemedicine Union (Association)), \\ Gocha Ghortlishvili (Researcher, Georgian Telemedicine Union (Association)), \\ Dodo Agladze (Researcher, Georgian Telemedicine Union (Association))
}

\begin{abstract}
Medical information system (MIS) is at the heart of information technology (IT) implementation policies in healthcare systems around the world. Different architecture and application models of MIS are developed. Despite of obvious advantages and benefits, application of MIS in everyday practice is slow. On the background of analysis of the existing models of MIS in Georgia has been created a multi-user web-based approach. This article will present the architecture of the system and its application for cytology quality assurance programs. Five hundred Georgian language electronic medical records from the cervical screening activity illustrated by images were selected for quality assurance program. The primary goal of the MIS is patient management. However, the system can be used for quality assurance programs. The ideal of healthcare in the information age must be to create a situation where healthcare professionals spend more time creating knowledge from medical information and less time managing medical information. The application of easily available and adaptable technology and improvement of the infrastructure conditions is the basis for eHealth applications. The MIS is perspective and actual technology solution. It can be used for cytology quality assurance programs.
\end{abstract}

Keywords - Digital images; Medical information system; Quality assurance program.

\section{INTRODUCTION}

There is a very clear need for the expanded application of information technology (IT) in healthcare. Clinical workflow still depends largely on manual, paper-based medical record systems, which is economically inefficient and produces significant variances in medical outcomes. Medical information system (MIS) is at the heart of IT implementation strategy in healthcare systems around the world. This is a comprehensive solution that automates the clinical, administrative, and supply-chain functions. It enables healthcare providers to improve their operational effectiveness, to reduce costs and medical errors, and to enhance quality of care. The aim of MIS was and is as simple as relevant: to contribute to and ensure a high-quality, efficient patient care. The relevance of "good" MIS for high-level quality of care is obvious. Without having appropriate access to relevant data, practically no decisions on diagnostic, therapeutic, or other procedures can be made [1].

The existing models and protocols of MIS are based on implementation of profiles such as HL7 and DICOM. Despite of obvious advantages and benefits, practical application of MIS in everyday practice of healthcare organization is slow. Research and development projects are ongoing in several countries around the world to develop MIS: examples include Canada, Australia, England, the United States, and Finland [2]. MIS is used primarily for setting objectives and planning patient care, documenting the delivery of care, and accessing the outcomes of care. It includes information regarding patient needs during episodes of care provided by different healthcare professionals [3]. The amount and quality of information available to healthcare professionals in patient care has an impact on the outcomes of patient care and the continuity of care. The information included in MIS has several different functions in the decision-making process in patient care. It also supports decision making in management and in health policy. MIS is effectively used for creation, editing and maintenance of the electronic medical records (EMR). In many countries, it is tedious to obtain copies of medical records, which makes it difficult to realize second opinion consultations [4]. EMR created by MIS can be maintained, shared with colleagues, and can support the maintenance of ac-curate and complete medical records [5]. An EMR can take multiple forms: an independent software application running on a single computer; a web service belonging to a single organization; a general web service as a platform with which to collect different types of medical information; or a USBbased EMR [6], [7]. MIS has been launched in Georgia. Its primary goal is patient management and creation of EMR. However, the system is also targeted at creating a unified information space in the framework of the wider medical organization. It can be successfully used for implementation of telemedicine consultations too. Telemedicine services are rapidly becoming an integral part in many hospitals and clinics around the world [8], [9]. In many programs, image based second opinion consultations account for over $50 \%$ of all eHealth activities. The goal of this article is to present the

\footnotetext{
* Corresponding author.

E-mail: ekldiashvili@newvision.ge
} 
application of MIS and digital images for online cytology quality assurance programs under the conditions prevailing in Georgia.

Georgia is not lagging far behind in the field of digital imaging and online quality assurance pro-grams. Perspectives and strategies of digital imaging are currently evolving, as emerging requirements would allow self-sustainable large scale exploitation while recent technological developments are available to support integrated and cost-effective solutions to such requirements. However, as far as we know digital images have proceeded to large scale exploitation in healthcare, even after successful technological demonstration phases [10]-[14]. Digital imaging is the important and powerful approach for ensuring the safe medical care.

Use of digital imaging approach is appearing to have many advantages over conventional light microscopy. The International Union Against Cancer (UICC) has estimated that at least in $5 \%$ to $10 \%$ of cancer cases a pathologist need consultation during routine work because of uncertainty [15]. Sending glass slides or paraffin blocks by mail or courier for experts in the field, is a time consuming way especially in critical specimens for pathologists working alone in distant hospitals with no facilities for intradepartmental consultation [16]. Besides, the probability of loss and damage are always pre-sent. Today, digital image based consultations in the forms of static and dynamic series seems to be the basic solution for this major problem. Conventional pathology and cytology with glass slide has many limitations. For example they may be easily broken, their stain is unstable and could fade with time, the tissue mount can bubble and dry out and finally certain procedure such as fluorescent stains are not stable more than few days. In this situation it seems that the best replacement for conventional slide pathology and cytology is image based diagnostic, which never change in appearance as long as the data integrity is maintained. However, in spite of mentioned points digital imaging in Georgia isn't popular. It is used only for consultation in limited healthcare centers and only in the form of static telepathology (sending of images and medical data to expert via email) because of the limitations in equipment, bandwidth of internet connections, low foreign language ability and computer literacy among healthcare professionals.

This article presents the data of a pilot study. The study aimed usage of MIS as the technological background for the digital image based cytology quality assurance program organization and implementation.

\section{METHODS}

The MIS has been created with .Net technology and SQL database architecture. It involves a multi-user web-based approach. This ensures local (intranet) and remote (internet) access of the system and management of databases. .Net technology can be installed on computers running Microsoft Windows operating systems. It includes a large library of coded solutions to common programming problems. .Net technology is a Microsoft offering and is intended for usage by most new applications created for the Windows platform.
Version 3.0 of the .Net technology included with Windows Server 2008 has been used for creation of MIS. The MIS is object-oriented software. It is realizing, client-server concept. Its architecture provides a secure, robust, and extensible system for managing multiple medical terminals within a centralized repository. The MIS has a flexible architecture that can run on numerous combinations. The recommended serveroperating requirement is Windows Server 2003. Hardware requirements are the following: memory $-1 \mathrm{~GB}$; disk space 1 GB. Internet Explorer 6.0+ and/or Mozilla Firefox 2.0+ can be used as client browsers.

The MIS consists of three key modules: 1. Administration and configuration module; 2 . Working module for medical personnel; 3. Reporting module.

The administration and configuration module is dedicated for setting up users' basic rights. It allows users registration or blocking, defining, and configuring of their rights. All medical forms (consultation, clinical investigation, diagnosis, prescription, treatment, etc.) are generated by this module. It is a database of staff too. Each employee is provided by a unique code, alongside with gathering biographical and professional data.

Using the working module, electronic medical record (EMR) can be generated, edited and updated by medical personnel. At generation moment, a unique code is given to EMR. It consists of text and multimedia files - images, video, and voice. Planning of patient visits and schedule of staff are also implemented by this module.

EMR can be fully or partially exported by the reporting module in various file formats, including pdf, rtf, and jpg. This module also implements statistical analysis of medical data (patient's age, sex, diagnosis, date of investigation, treatment parameters, etc.). It can be used for quality control of medical services.

The MIS has been used for cervical cancer screening program. The target group of this screening program were 18 to 65 years old women attending four women's consultation clinics in Tbilisi, Georgia, with chief complaints of vaginal discharge and vaginal bleeding. Totally one thousand fifty patients of cervical cancer screening program were registered in the MIS during 18 months, the EMRs of the patients contain text data and digital images of the cervical smears. The cervical smears were collected by gynecologists using cervical brushes, plated on microscopy slides, fixed with $95 \%$ ethanol and transported to the laboratory. After transportation of the slides to the laboratory, to reduce the level of air-drying artifacts on the slides, they were rehydrated by immersion in normal saline for five minutes and then fixed in $95 \%$ ethanol. Smears were stained using the standard Papanicolaou technique. The adequacy of each of the paired slides was determined separately. All slides were examined and assessed for both cytology and staining quality, with results reported using the Bethesda System. The images were captured by using a 2.0 USB digital eyepiece microscope camera with resolution 3.0 MP after routine cytology investigation. The images resolution was $2048 \times 1536$ pixels. The average number of images per case was five (462 cases, $92.4 \%$ ). Each 
series of images per case began with a general view, followed by higher magnification of diagnostically interesting and actual areas. These areas were chosen by a cytologist. The images were taken by a laboratory personnel trained in using the camera. The images were stored in a personal computer. They were adjusted by using Adobe Photoshop and uploaded to the corresponding EMR. The adjustment mainly aimed improvement of contrast and brightness. This manipulation has been done by laboratory personnel.

Five hundred EMRs of cervical screening activity have been randomly selected for online cytology quality assurance program. The randomization has been done by application of the Research Randomizer. This is a free service offered to students and researchers interested in conducting random assignment and random sampling. The service is available at www.randomizer.org. The participants of online cytology quality assurance program were registered as users at MIS, they accessed the sys-tem and EMRs through internet. For simplicity reason the registration numbers of selected for online cytology quality assurance program EMRs were listed at home page of MIS.

\section{RESULTS}

From January 2015 to October 2015, 500 cases of cervical cancer screening program were used for online cytology quality assurance program organization and implementation through application of MIS. EMRs of these cases included text data (the patient specific data - name, address and etc., has been omitted) and cervical smears digital images. Cervical smears were conventional Pap-smears. The slides were photographed with a 2.0 USB digital eyepiece microscope camera with a resolution of $3.0 \mathrm{MP}$. The images had a resolution of $2048 \times 1536$ pixels. The average number of images per case was five (462 cases, $92.4 \%$ ). Each series of images per case began with a general view, followed by higher magnification of diagnostically interesting and actual areas. The cytologist selected these areas. The images were captured by laboratory personnel trained in using the camera, and they used Adobe Photoshop to adjust image contrast and brightness.

Four Georgian certified cytologists with experience of participation in image based second opinion consultations were selected as participants for online cytology quality assurance program. They have been registered as users at MIS and accessed system and EMRs through internet. The registration numbers of selected for online cytology quality assurance program EMRs were listed at home page of MIS.

For digital images quality control, each participant of quality assurance program subjectively assessed the image quality. Image sharpness and quality were rated using a 4level scale: 1 - excellent; 2 - good; 3 - fair; 4 - poor. The average number of participants per quality assurance case was three (470 cases, $94.0 \%$ ). In 428 cases $(85.6 \%$ ) first reply was made in less than 6 hours. In 485 cases $(97.0 \%)$ the primary diagnosis has been matched during online cytology quality assurance program. In 5 cases $(1.0 \%)$ the different diagnosis have been reported. In 10 cases $(2.0 \%)$ the images were re-ported as of poor quality and insufficient for online cytology quality assurance program.

Overall, $96.0 \%$ of the cases were rated as having excellent or good image sharpness and contrast, with $4.0 \%$ being rated as fair or poor. With respect to image color, $97.0 \%$ of the images were rated as excellent or good, with only $3.0 \%$ being rated as fair or poor. There was a high positive correlation ( $r=0.88$ ) between color, sharpness, and contrast ratings. Images with excellent or good ratings generally received excellent or good color ratings. There were relatively low correlations between color $(r=0.25)$ and sharpness/contrast $(r=0.30)$ ratings the decision confidence values.

\section{DISCUSSION}

Online quality assurance programs with usage of digital images can be used in many areas of pathology. It is practical and cost-effective and provide many advantages over traditional pathology practices. Digital imaging also is the first step toward opening the door to many future applications and improved diagnostic, educational and quality assurance activities. Most of the studies on digital image based diagnostic have been confined to the study of breast aspiration biopsies. There is 1 published study on telecytology using video microscopy in cervical smears [17]. However, in that study, video microscopy was used without using digitalization and image transmission. Our study is the first to examine the usage of the medical information system and digital imaging for quality assurance programs organization and implementation.

There are many advantages of using the MIS for online cytology quality assurance programs. The major advantage is the rapid turnaround time. Access of EMR through the internet undoubtedly is faster than the conventional method of circulating glass slides. The use of EMRs and digital images also ensures the assessment of the identical medical data, avoiding the problem posed by difference in field selection. Thus, digital image based cytology quality assurance program realized by application of the MIS circumvents the problem of field selection and assesses interpretation. Cost savings is serious and obvious advantage. Digital images based quality assurance programs organized by application of MIS reduce the expenses of postal or courier slide circulation. Easy and continuous access to the case material should be achieved through their storage in the MIS. After the cycle of the quality assurance program the EMRs are still available for reference and other healthcare purposes.

Three factors can affect the quality assurance program on digital images: adequate and appropriate sampling (field selection, number and microscopic magnification of images), image resolution and color quality. Cytologic diagnosis is based on cytologic features such as nuclear/cytoplasmic ration, chromatin pattern, nuclear pleomorphism, and cellular arrangement on a limited number of microscopic fields. In digital imaging, one of the primary problems has been field selection (sampling error). Accuracy for digital imaging has varied between $85 \%$ and $100 \%$. Insufficient or inadequate sampling of images is the most common reason for low 
diagnostic accuracy. However, this can be minimized when an experienced pathologist/cytologist who is familiar with the technology and trained to recognize and identify highly informative fields is available for field selection [18]. With the development of total slide digitalization systems, this problem can be circumvented [19].

In the present study has been shown, that magnification at $\times 100$ was necessary to identify the back-ground and architecture of cell clusters. However, $\times 40$ magnification was too low a resolution to permit interpretation of the digital images. Resolution is the ability to show fine details of visual information. The higher the resolution, the more visual information present. Low resolution and color depth were limiting factors in digital images of the past. However, the resolution and color depth of current digital cameras and especially digital eyepiece microscope cameras are more than adequate to make most cytologic diagnoses [20], [21]. Compression of the image file is another important factor that affects the quality of digital image, and lost visual information can never be recovered after image compression. JPEG is by far the most commonly used method of compressing image files. In the present study with high resolution $(2048 \times 1536$ pixels) JPEG image files could be interpreted accurately and showed excellent cellular details for online cytology quality assurance program.

\section{V.CONCLUSION}

Perspectives and strategies of digital imaging are currently evolving. The emerging operative requirements would allow self-sustainable large-scale exploitation, but recent technological developments are available to support integrated and cost-effective solutions to such requirements. However, after successful technological demonstration phases, only a few digital imaging applications have proceeded to large-scale exploitation. Digital imaging approach is of decisive importance for ensuring of safe medical care. Use of digital images for cytology quality assurance program has many advantages over conventional light microscopy. Sending glass slides by mail or courier to experts in the field is time consuming especially in critical specimens for cytologists working alone in distant hospitals with no facilities for intradepartmental consultation [22]. Further, the probability of loss and damage is always present.

We concluded, that MIS can be successfully used for creation of EMRs and implementation of online cytology quality assurance program. It has been revealed that the MIS has yielded significant benefits: a) Easy access to medical data; b) Structured information; c) Safe and secure second opinion consultations. The system is a very useful and easy for use tool. It has been revealed, that the MIS can be easily and effectively applied for online quality assurance programs in cytology. We concluded that the MIS is a useful and applicable tool for routine medical practice. It significantly increases knowledge exchange and thereby ensures a better medical service.

\section{EXTENDED SUMMARY}

Introduction - Medical information system (MIS) is at the heart of information technology (IT) implementation policies in healthcare systems around the world. Different architecture and application models of MIS are developed. Despite of obvious advantages and benefits, application of MIS in everyday practice is slow. Objective - On the background of analysis of the existing models of MIS in Georgia has been created a multi-user web-based approach. This article will present the architecture of the system and its application for cytology quality assurance programs. Methods - The MIS has been created with .Net technology and SQL database architecture. It realizes local (intranet) and remote (internet) access to the system and management of databases. The MIS can be successfully used for medical data registration and management as well as for creation, editing and maintenance of the electronic medical records (EMR). Five hundred Georgian language electronic medical records from the cervical screening activity illustrated by images were selected for quality assurance program. Results - The primary goal of the MIS is patient management. However, the system can be used for quality assurance programs. Discussion - The ideal of healthcare in the information age must be to create a situation where healthcare professionals spend more time creating knowledge from medical information and less time managing medical information. The application of easily available and adaptable technology and improvement of the infrastructure conditions is the basis for eHealth applications. Conclusion the MIS is perspective and actual technology solution. It can be used for cytology quality assurance programs.

\section{ACKNOWLEDGMENT}

The study has been performed during implementation of the project 'Morphology Online Quality Assurance Program' (\#31/05). This project was supported by Shota Rustaveli National Science Foundation.

\section{VIII.CONFLICT OF INTEREST}

No conflict of interest in connection with this article exists. No competing financial interests exist.

\section{REFERENCES}

[1] S. Clamp and J. Keen, "Electronic health records: Is the evidence base any use?," Medical Informatics and the Internet in Medicine, vol. 32, no. 1, pp. 5-10, Jan. 2007. https://doi.org/10.1080/14639230601097903

[2] K. Hayrinen, K. Saranto, and P. Nykanen, "Definition, structure, content, use and impacts of electronic health records: A review of the research literature," International Journal of Medical Informatics, vol. 77, $\quad$ no. 5, $\quad$ pp. 291-304, 2008. https://doi.org/10.1016/j.ijmedinf.2007.09.001

[3] A. M. van Ginneken, "The computerized patient record: balancing effort and benefit," International Journal of Medical Informatics, vol. 65, no. 2, pp. 97-119, Jun. 2002. https://doi.org/10.1016/s13865056(02)00007-2

[4] L. S. Liu, P. C. Shih, and G. R. Hayes, "Barriers to the adoption and use of personal health record systems," Proceedings of the 2011 iConference on-iConference '11, 2011. https://doi.org/10.1145/1940761.1940811 
[5] US Department of Health and Human Services, Office for Civil Rights 2008, Dec. 15. Personal Health Records and the HIPAA Privacy Rule. [Online].Available:

http://www.hhs.gov/ocr/privacy/hipaa/understanding/special/healthit/phr s.pdf [Accessed 2014-05-29]

[6] J. Bonander and S. Gates, "Public Health in an Era of Personal Health Records: Opportunities for Innovation and New Partnerships," Journal of Medical Internet Research, vol. 12, no. 3, p. e33, Aug. 2010. https://doi.org/10.2196/jmir.1346

[7] D. C. Kaelber, A. K. Jha, D. Johnston, B. Middleton, and D. W. Bates, "A Research Agenda for Personal Health Records (PHRs)," Journal of the American Medical Informatics Association, vol. 15, no. 6, pp. 729 736, Nov. 2008. https://doi.org/10.1197/jamia.m2547

[8] G. Heidenreich and B. Blobel, "IT-Standards für telemedizinische Anwendungen," Bundesgesundheitsblatt - Gesundheitsforschung Gesundheitsschutz, vol. 52, no. 3, pp. 316-323, Feb. 2009. https://doi.org/10.1007/s00103-009-0788-6

[9] B. Harnett, "Telemedicine systems and telecommunications," Journal of Telemedicine and Telecare, vol. 12, no. 1, pp. 4-15, Jan. 2006. https://doi.org/10.1258/135763306775321416

[10] K. J. Kaplan, J. R. Burgess, G. D. Sandberg, C. P. Myers, T. R. Bigott, and R. B. Greenspan, "Use of Robotic Telepathology for Frozen-Section Diagnosis: A Retrospective Trial of a Telepathology System for Intraoperative Consultation," Modern Pathology, vol. 15, no. 11, pp. 1197-1204, Nov. 2002 https://doi.org/10.1097/01.mp.0000033928.11585.42

[11] K. Brauchli and M. Oberholzer, "Comparison of telepathology services," Journal of Telemedicine and Telecare, vol. 10, no. 5, pp. 307308, Oct. 2004. https://doi.org/10.1258/1357633042026350

[12] A. Abdirad, B. Sarrafpour, and S. Ghaderi-Sohi, "Static telepathology in cancer institute of Tehran university: Report of the first academic experience in Iran," Diagnostic Pathology, vol. 1, no. 1, p. 33, 2006 https://doi.org/10.1186/1746-1596-1-33

[13] K. Brauchli, H. Oberli, N. Hurwitz, K.-D. Kunze, G. Haroske, G. Jundt, G. Stauch, L. Banach, M. Wirdnam, M. Mihatsch, and M. Oberholzer, "Diagnostic telepathology: long-term experience of a single institution," Virchows Archiv, vol. 444, no. 5, pp. 403-409, May 2004 https://doi.org/10.1007/s00428-004-0980-x

[14] B. H. Williams, F. G. Mullick, D. R. Butler, R. F. Herring, and T. J. O'Leary, "Clinical evaluation of an international static image-based telepathology service," Human Pathology, vol. 32, no. 12, pp. 13091317, Dec. 2001. https://doi.org/10.1053/hupa.2001.29649

[15] Kldiashvili E, et al. "Application of Digital Imaging for Cytopathology under Conditions of Georgia" AIMS Medical Science, 2015, 2(3): 186199. https://doi.org/10.3934/medsci.2015.3.186

[16] P. N. Furness, "The use of digital images in pathology," The journal of Pathology, vol. 183, issue 3, pp. 15-24, Nov. 1997. https://doi.org/10.1002/(sici)1096-9896(199711)183:3<253::aidpath927>3.0.co;2-p

[17] S. S. Raab, M. Sue Zaleski, P. A. Thomas, T. H. Niemann, C. Isacson, and C. S. Jensen, "Telecytology:Diagnostic Accuracy in CervicalVaginal Smears," American Journal of Clinical Pathology, vol. 105, no. 5, pp. 599-603, May 1996. https://doi.org/10.1093/ajcp/105.5.599

[18] D. S. Weinberg, F. A. Allaert, P. Dusserre, F. Drouot, B. Retailliau, W. R. Welch, J. Longtine, G. Brodsky, R. Folkerth, and M. Doolittle, "Telepathology diagnosis by means of digital still images: An international validation study," Human Pathology, vol. 27, no. 2, pp 111-118, Feb. 1996. https://doi.org/10.1016/s0046-8177(96)90363-9

[19] F. J. W.-M. Leong and J. O. McGee, "Automated complete slide digitization: a medium for simultaneous viewing by multiple pathologists," The Journal of Pathology, vol. 195, no. 4, pp. 508-514, 2001. https://doi.org/10.1002/path. 972

[20] B. E. Halliday, A. Bhattacharyya, A. R. Graham, J. R. Davis, S. A. Leavitt, R. B. Nagle, W. J. Mclaughlin, R. A. Rivas, R. Martinez, E. A Krupinski, and R. S. Weinstein, "Diagnostic accuracy of an international static-imaging telepathology consultation service," Human Pathology, vol. 28, no. 1, pp. 17-21, Jan. 1997. https://doi.org/10.1016/s00468177(97)90273-2

[21] M. H. Weinstein and J. I. Epstein, "Telepathology diagnosis of prostate needle biopsies," Human Pathology, vol. 28, no. 1, pp. 22-29, Jan. 1997. https://doi.org/10.1016/s0046-8177(97)90274-4

[22] C. A. Wells and C. Sowter, "Telepathology: A diagnostic tool for the millennium?" The journal of Pathology, vol. 191, issue 1, pp. 1-7, May 2000. https://doi.org/10.1002/(sici)1096-9896(200005)191:1<1::aidpath624>3.0.co;2-s
Ekaterine Kldiashvili received a MSc in Biology - Cell and Molecular Biology in 1995 and a Ph.D. in Histology, Cytology and Embryology in 2003 from the Tbilisi State University. She is a member of academic staff of the New Vision University: Associate Professor from 2013-2016; Professor since 2016. She is Executive Director of Georgian Telemedicine Union (Association), manages projects funded by Rustaveli Science Foundation and 700 for Science, the whole activity of the Georgian Telemedicine Union (Association) and also the creation of eHealth network in Georgia. From 2007 to 2011, she was Principal Investigator of NATO Networking Infrastructure and BSEC projects, a participant of COST Action IC0604 (Telepathology Network in Europe: EURO-TELEPATH. Prior to joining the Georgian Telemedicine Union (Association) she worked at the Department of Pathology of Central Clinic of the Tbilisi State Medical University, establishing and leading the electron microscopy laboratory. Ekaterine is a certified specialist of laboratory medicine with an experience in setting up, developing and managing clinical laboratories ("NeoClinic", 2009-2010; "NeoLab", 2010-2012; "TestDiagnostics", 2013-2014; Center of Molecular Pathology and Early Diagnostics of Ltd "New Vision University Hospital", 2015), preparing and realizing (in-person and online) educational courses on actual topics of laboratory medicine (14 CME programs, 2006-2008). She is leading the PhD program in medicine at the New Vision University. Ekaterine was trained in cytological diagnosis of malignant tumors at the Department of Oncology of the Tbilisi State Medical Academy (2002, Tbilisi, Georgia) and Gynecological Cytology Diagnostics by application of the Liquid Based Cytology Method at company "HOLOGIC" (2014, Rome, Italy). She presented the activity and projects of the Georgian Telemedicine Union (Association) at Med-e-Tel (2004, 2005, 2006, 2008, 2010), Telemedicine Week 2005 (Institute of Tropical Medicine, Antwerp, Belgium), II International Seminar on Telemedicine (Ukraine, 2006), Telemedicine Conference in South Africa (2006), the 2nd International Conference and Exhibition on Pathology (2013, USA), The 2014 Pathology Congress (UK), Pathology 2015 (UK). Ekaterine Kldiashvili has published about 35 articles and been an editor of a book.

Andrejs Romānovs, Dr.sc.ing., MBA, Associate Professor and Senior Researcher at the Riga Technical University (Latvia). His professional interests include modeling and design of management and industrial information systems, IT governance, cybersecurity and IT risk management, information technologies and systems for health care, e-commerce, logistics, as well as education in these areas.

$\mathrm{He}$ is an expert of the Latvian Council of Science in the field of IT; member of the Council of RTU ITI; senior member of the IEEE, Chair of IEEE R8 MDSC, Chair of IEEE Latvia Section; member and academic advocate at ISACA, member of LSS. He has published more than 70 textbooks and scientific papers in the field of Information Technology, has organized and participated in over 50 international scientific conferences, as well as in numerous scientific and R\&D projects.

Address: IT Institute, Riga Technical University, 1 Kalku Str., Riga, LV-1658, Latvia.

E-mail: andrejs.romanovs@rtu.lv 